Copyright (c) the authors 2018. Meeting abstract published in European Respiratory Journal 2018 52: Suppl. 62, OA3311. doi: 10.1183/13993003.congress-2018.0A3311. This is an ERS International Congress abstract. No full-text version is available.

\title{
Using a Bayesian approach and external validation to predict persistent asthma at the age of 10 and 20 years in general and high-risk populations
}

\author{
Silvia Colicino, Cosetta Minelli, Alexandra Lewin, Stephen Turner, Angela Simpson, Hasan Ars \\ had, Graham Roberts, John Henderson, Adnan Custovic, Paul Cullinan
}

Several studies have established tools to forecast asthma but their clinical usefulness and reproducibility in external populations is limited. We developed and validated models for asthma prediction in school age and early adulthood using information collected in pre-school age. We used data from 5 UK cohorts and studied 2 groups: (1) all children to identify common patterns; and (2) children with wheezing between 2 and 5 years considered at high-risk of asthma. Two cohorts with similar recruitment criteria (training data) were used to develop Bayesian predictive models; the remaining studies (validation data) were employed to assess their performance by calibration and discrimination measures. We considered 39 potential predictors collected within the first 5 years of life including perinatal information, respiratory symptoms, environmental exposures and family-history. We defined asthma by the presence of current wheeze and asthma treatment at ages 10 and 20 years. We included 14,614 and 1,360 children for the two groups in the training data, and validated models on 4,564 and 569 children from the validation data. Asthma prevalence among cohorts ranged from 8$19 \%$ and $24-41 \%$ in the two groups, respectively. Frequency of wheezing, dust mite sensitisation and doctor's diagnosis of eczema were important predictors for asthma in both groups. Our methods showed good performance with an accuracy of $80 \%$; sensitivity and positive predictive value were $63 \%$ and $33 \%$ in general population and $81 \%$ and $60 \%$ in high-risk group. While for the general population our predictive model performed similarly to other existing tools, for the high-risk population our model provided better predictive ability than available externally validated tools. 